Vietnam Journal of Mechanics, VAST, Vol. 26, 2004, No2 (111-121)

\title{
THE METHOD OF DETERMINING INTERNAL FORCES AT ANY CROSS SECTION OF LINKS IN MECHANISMS
}

\author{
Do Sanh, Do Dang KHOA \\ Hanoi University of Technology
}

\begin{abstract}
In the paper it is introduced a method of determining internal forces at any cross section of the links of mechanisms. As known, so far it is used the method of D'Alembert, which consists of two steps, the determination of the acceleration states of links and the establishment of the equilibrium equations for the set of forces including the forces of inertia and the internal forces at the cross section. A. I. Lurie proposed a method of analytical mechanics for this problem. Its concept is to make a new system called the released one by cutting the link at a cross section under consideration and adding some coordinates. Only one condition putting restriction on the released system is the additional coordinates must equal zero. Under this restriction the new created system is coincided to the original one. This restriction is equivalent to put the mechanical constraints, whose reaction forces are the components of internal forces at the cross section under consideration. It is necessary emphasize that the Lurie's method is convenient only for opened loops, but is not applied for closed ones. Moreover, the Lagrange's multiplier equations applied by A. I. Lurie are unsuitable. In this paper it is presented the generalized Lurie's method, which is applied for the opened and closed loops by using the Principle of Compatibility.
\end{abstract}

\section{Introduction}

The problem of determining internal forces at any cross section of links of mechanism is very important because in modern dynamics the cross section of links is designed increasingly small while their angular velocities are more and more larger.

In the past, for determining the components of internal forces (the tensible, shearing force and bending moment) the D'Alembert's method is applied. For doing this, first it is necessary to determine the acceleration state of links and next to write the equilibrium equations for the set of forces in included the forces of inertia.

Lurie A. I. proposed the method for simultaneously determining the acceleration state and components of internal forces [8].

The idea of the Lurie's method is to make the system called the released one by cutting the link at a cross section and three new coordinates are introduced. Only one condition putting restriction on the released system: the addition coordinates must equal zero. By annulling addition coordinates we get the equations, which are treated as constraint equations. The reaction forces of these constraints just are the components of internal forces at the cross section under consideration. The idea of the Lurie's method is excellent. However this method is not applied for closed loops due to hardly choosing the independent coordinates.

In this paper by using the Lurie's method together the application of the Principle of Compatibility the components of internal forces at any cross section of links of mechanism are determined. 


\section{The method of determining the components of internal forces at any cross section of links}

Let us consider a closed plane loop of $n$ degree of freedom (Fig. 1). The number of independent coordinates of this system is equal to $n$.

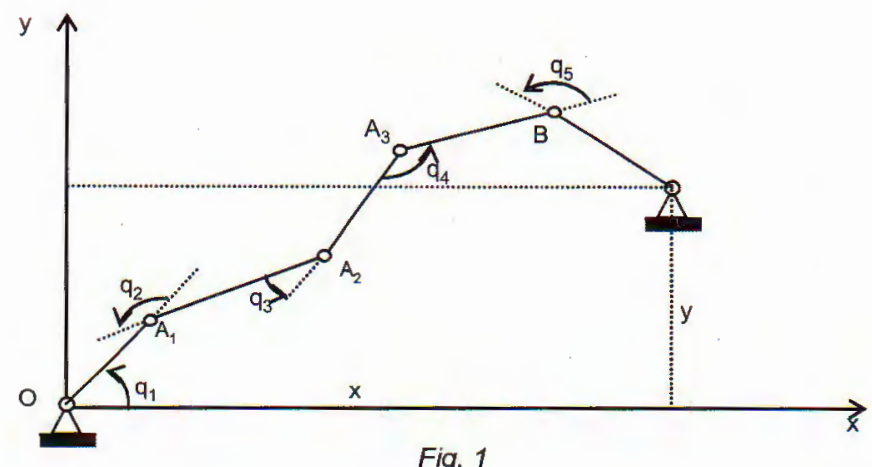

Fig. 1

However, the choice of independent coordinates is uncomfortable for dynamic investigation, for example, in the calculation of kinetic energy and etc,...

Let us choose the coordinates $q_{j}(j=\overline{1, m})$. Of course, between the chosen coordinates $q_{j}(j=\overline{1, m}>n)$ there exist the constraint equations:

$$
f_{\alpha}\left(q_{1}, q_{2}, \ldots, q_{m}\right)=0, \quad r=m-n, \quad \alpha=\overline{1, r} .
$$

In order to apply the Lurie's method it is necessary to release the hinge $B$ and to put the components of $X_{B}, Y_{B}$ reaction forces, which are treated as the applied forces to mechanism.

By such a way we get an opened loop. The number of coordinates of this system is equal to $(m+2)$, that are $\left\{q_{1}, q_{2}, \ldots, q_{m}, x, y\right\}$, where $x, y$ are coordinates of the hinge $B$. The obtained system is restricted by the constraints (2.1) and the constraints of the form.

$$
\begin{aligned}
& x-g_{1}\left(q_{1}, q_{2}, \ldots, q_{m}\right)=0, \\
& y-g_{2}\left(q_{1}, q_{2}, \ldots, q_{m}\right)=0 .
\end{aligned}
$$

These constraints belong to the conventional constraints (the programmed constrains) $[1,2,5,7]$.

Let us apply the Lurie's method for the opened system obtained. By cutting the link under consideration at the defined section. By such a way the system is separated into two parts (Fig. 2). In order to define the position of the system three coordinates $u, v, w$ are introduced, which are called the addition coordinates.

The cutted system will be identical with the original system if the following conditions are realised

$$
u=0, \quad v=0, \quad w=0 .
$$

The restrictions (2.3) are treated as constraints, but their reaction forces just are the components of internal forces at the cross section under consideration, which one after another are the tensible, shearing forces and bending moment. 


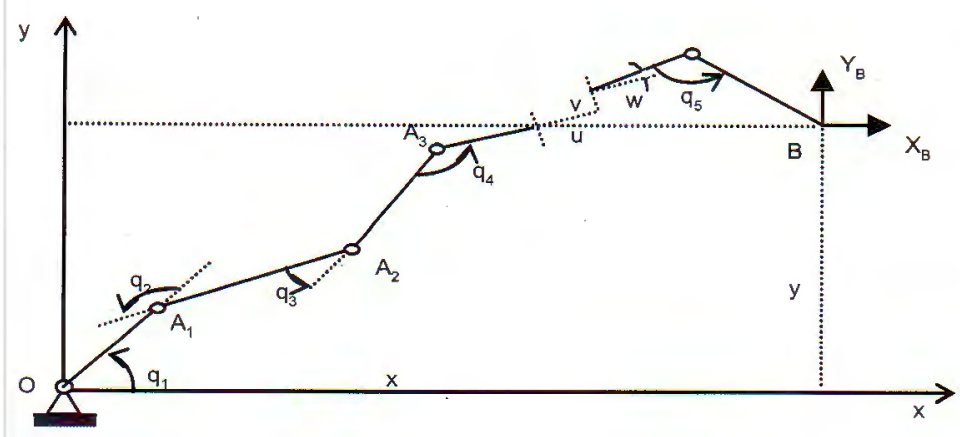

Fig. 2

Denote the kinetic energy of the cutted system and the generalised forces by $T, Q_{j}(j=$ $\overline{1, m}), Q_{x}, Q_{y}, Q_{u}, Q_{v}, Q_{w}$ respectively, but the reaction forces by $R_{j}(j=\overline{1, m}), R_{x}, R_{y}$, $R_{u}, R_{v}, R_{w}$ respectively.

By following the Principle of Compatibility [3], we write the equations of the cutted system in the form

$$
\begin{aligned}
& \frac{d}{d t} \frac{\partial T^{0}}{\partial \dot{q}_{j}}-\frac{\partial T^{0}}{\partial q_{j}}=Q_{j}^{0}+R_{j} \\
& \frac{d}{d t} \frac{\partial T^{0}}{\partial \dot{x}}-\frac{\partial T^{0}}{\partial x}=Q_{x}^{0}+R_{x} \\
& \frac{d}{d t} \frac{\partial T^{0}}{\partial \dot{y}}-\frac{\partial T^{0}}{\partial y}=Q_{y}^{0}+R_{y} \\
& \frac{d}{d t}\left(\frac{\partial T}{\partial \dot{u}}\right)^{0}-\left(\frac{\partial T}{\partial u}\right)^{0}=Q_{u}^{0}+R_{u} \\
& \frac{d}{d t}\left(\frac{\partial T}{\partial \dot{v}}\right)^{0}-\left(\frac{\partial T}{\partial v}\right)^{0}=Q_{v}^{0}+R_{v} \\
& \frac{d}{d t}\left(\frac{\partial T}{\partial \dot{w}}\right)^{0}-\left(\frac{\partial T}{\partial w}\right)^{0}=Q_{w}^{0}+R_{w}
\end{aligned}
$$

where the symbol ( $)^{0}$ means that the quantities $u, v, w$ in the bracket must be took the zero value.

The reaction forces $R_{j}(j=\overline{1, m}), R_{x}, R_{y}, R_{u}, R_{v}, R_{w}$ must satisfy the condition of ideality of constraints. For getting this condition let us choose the quasi-accelerations [3, $6,8]$, for example, $\ddot{q}_{i}(i=\overline{1, n}), x, y$ and write the expression of accelerations in terms of quasi-accelerations by using the given constraint equations, that are

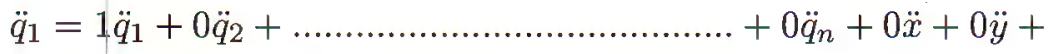

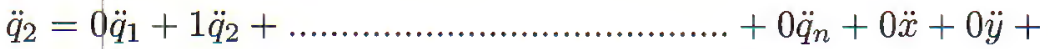

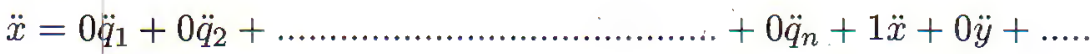

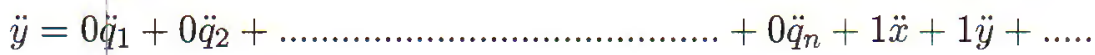




$$
\begin{aligned}
& \ddot{q}_{n+1}=k_{n+1,1} \ddot{q}_{1}+k_{n+1,2} \ddot{q}_{2}+\ldots+k_{n+1, n} \ddot{q}_{n}+k_{n+1}^{(x)} \ddot{x}+k_{n+1}^{(y)} \ddot{y}+\ldots . . \\
& \ddot{q}_{n+2}=k_{n+2,1} \ddot{q}_{1}+k_{n+2,2} \ddot{q}_{2}+\ldots+k_{n+2, n} \ddot{q}_{n}+k_{n+2}^{(x)} \ddot{x}+k_{n+2}^{(y)} \ddot{y}+\ldots . \\
& \ddot{q_{m}}=k_{m, 1} \ddot{q}_{1}+k_{m, 2} \ddot{q}_{2}+\ldots \ldots \ldots \ldots \ldots+k_{m, n} \ddot{q}_{n}+k_{m}^{(x)} \ddot{x}+k_{m}^{(y)} \ddot{y}+\ldots \ldots
\end{aligned}
$$

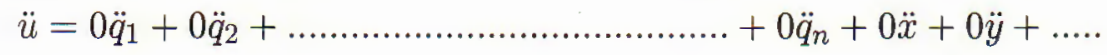

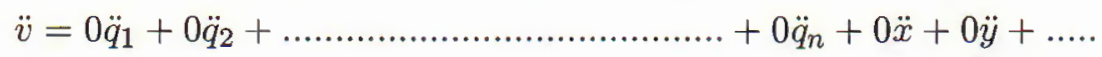

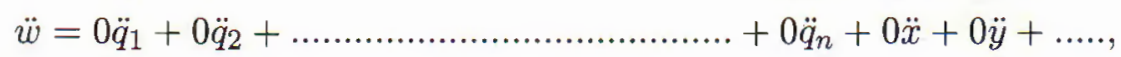

where the non-written terms do not contain accelerations, but the coefficients $k_{\alpha i}$ $(\alpha=\overline{n+1, m}, i=\overline{1, n}), k_{\alpha}^{(x)}, k_{\alpha}^{(y)}(\alpha=\overline{n+1, m})$ are the known functions of coordinates.

From here we get the matrix $\mathbf{D}$

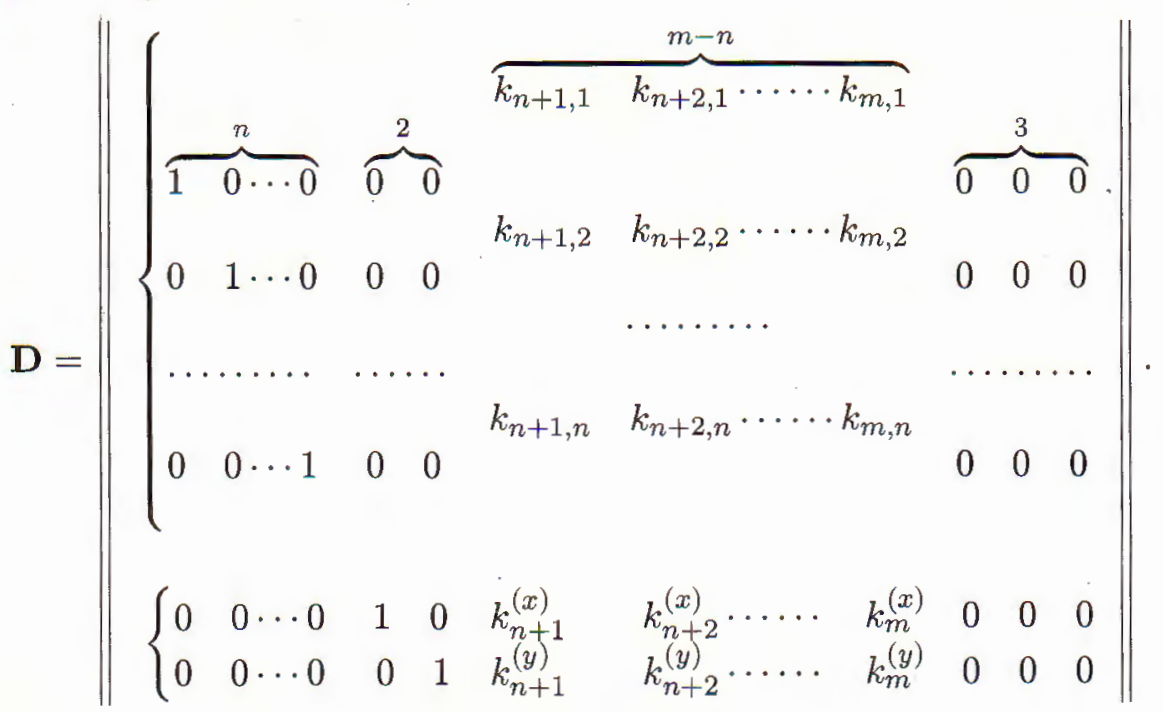

The condition of ideality of constraints is of the form

$$
\mathbf{D} \mathbf{R}=0
$$

where

$$
\mathbf{R}^{T}=\left\|R_{1} \cdots \cdots R_{m} \quad R_{x} \quad R_{y} \quad R_{u} \quad R_{v} \quad R_{w}\right\| .
$$

By (2.9) we obtain

$$
\begin{gathered}
R_{i}+\sum_{\alpha=n+1}^{m} k_{\alpha i} R_{\alpha}=0, \quad i=\overline{1, n} \\
R_{x}+\sum_{\alpha=n+1}^{m} k_{\alpha}^{(x)} R_{\alpha}=0 \\
R_{y}+\sum_{\alpha=n+1}^{m} k_{\alpha}^{(y)} R_{\alpha}=0 .
\end{gathered}
$$


In consideration of $(2.4),(2.5)$ and $(2.6)$, the equations (2.11) are written in the form:

$$
\frac{d}{d t} \cdot \frac{\partial T^{0}}{\partial \dot{q}_{i}}-\frac{\partial T^{0}}{\partial q_{i}}-Q_{i}^{0}+\sum_{\alpha=n+1}^{m} k_{\alpha i}^{0}\left(\frac{d}{d t} \frac{\partial T^{0}}{\partial \dot{q}_{\alpha}}-\frac{\partial T^{0}}{\partial q_{\alpha}}-Q_{\alpha}^{0}\right)=0, \quad i=\overline{1, n} .
$$

These equations together with the constraint equations (2.1) and given initial condition will describe the motion of the original system.

Let us now return to the equations (2.12). It is necessary to emphasize. The constraints (2.2) belong to the conventional constraints (the programme constraints). This means that their reaction forces are identical to zero $[1,7]$. Therefore we have

$$
\begin{gathered}
\sum_{\alpha=n+1}^{m} k_{\alpha}^{(x) 0}\left(\frac{d}{d t} \frac{\partial T^{0}}{\partial \dot{q}_{\alpha}}-\frac{\partial T^{0}}{\partial q_{\alpha}}-Q_{\alpha}^{0}\right)=0, \\
\sum_{\alpha=n+1}^{m} k_{\alpha}^{(y) 0}\left(\frac{d}{d t} \frac{\partial T^{0}}{\partial \dot{q}_{\alpha}}-\frac{\partial T^{0}}{\partial q_{\alpha}}-Q_{\alpha}^{0}\right)=0 .
\end{gathered}
$$

It is noticed that the components of the reaction force of the hinge $B$ belong to the applied forces. This means that these action forces are included in the expressions of the generalised forces $Q_{\alpha}^{0}(\alpha=\overline{n+1 ; m})$. Due to this from (2.14) it is to allow us is calculate the components $X_{B}$ and $Y_{B}$ of the reaction force of the hinge $\mathrm{B}$.

As known, the reaction forces of the constraints (2.3) just are the components of the internal force at the cross section.

These components are found from (2.6). That are

$$
\begin{aligned}
& R_{u}=\frac{d}{d t}\left(\frac{\partial T}{\partial \dot{u}}\right)^{0}-\left(\frac{\partial T}{\partial u}\right)^{0}-Q_{u}^{0} \\
& R_{v}=\frac{d}{d t}\left(\frac{\partial T}{\partial \dot{v}}\right)^{0}-\left(\frac{\partial T}{\partial v}\right)^{0}-Q_{v}^{0} \\
& R_{w}=\frac{d}{d t}\left(\frac{\partial T}{\partial \dot{w}}\right)^{0}-\left(\frac{\partial T}{\partial w}\right)^{0}-Q_{w}^{0}
\end{aligned}
$$

which one after another the tensible, shearing forces and bending moment at the indicated cross section.

\section{$3 \quad$ Examples}

Example 1. Let us determine the bending moment at the cross section located at the distance a with respect to the end $A$ of the link $A B$ of the slider-crank mechanism. The crank moves under action of the moment $\mathrm{M}$ of the motor, but the slider-the force $\vec{F}$. The length of the crank and transmission link are $r$ and $l$ respectively. For raison of simplicity the mass of the transmission link and the friction are neglected. The mass center of the crank is coincided with the axis of rotation, but the mass of the slider is of $m$ [Fig. 3].

First we make the free system by applying the axioms of constraints for the slider B by releasing it from slide-way. The reaction force from the slide-way acting on the slider is denoted by $\vec{N}$, which is included in the applied forces acting on the system. By such a 
way the applied forces acting on the system consist of $M, \vec{F}, \vec{P}$ and $\vec{N}$, where $\vec{P}$ is the weight of the slider, $J_{0}$-the moment of inertia of the crank about the axis 0 .

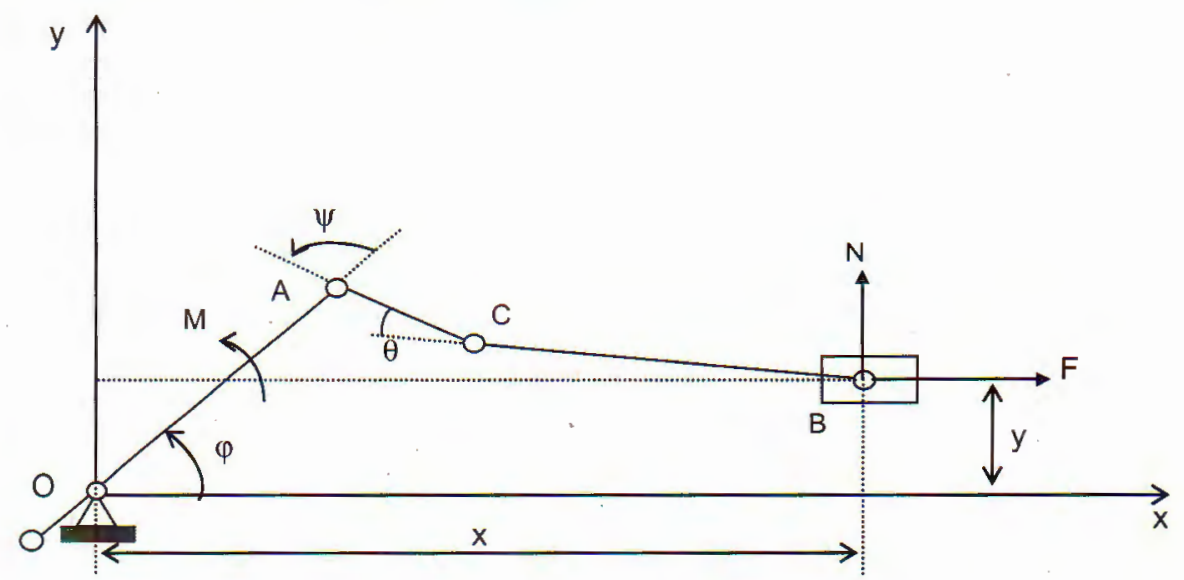

Fig. 3

In order to calculate the bending moment at the indicated section let us to make the cutted system and the generalised coordinates $\varphi, \psi, \theta, y$ are chosen as in Fig. 3 . The addition coordinate is $\theta$. The constraint equations are of the form

$$
\begin{aligned}
& f_{1} \equiv r_{1} s \varphi-a s(\varphi+\psi)-(l-a) s(\varphi+\psi+\theta)-y=0, \\
& f_{2} \equiv \theta=0 .
\end{aligned}
$$

Here and afterwards, the following symbols are used: $\cos \varphi=c \varphi, \sin \varphi=s \varphi$.

First let us calculate the kinetic energy of the system, which is of the form

$$
T=\frac{1}{2} J_{0} \dot{\varphi}^{2}+\frac{1}{2} m\left(\dot{x}^{2}+\dot{y}^{2}\right) \text {. }
$$

The coordinates $x, y$ of the slider have the expressions

$$
\begin{aligned}
& x=\operatorname{rc} \varphi-a c(\varphi+\psi)-(l-a) c(\varphi+\psi+\theta), \\
& y=r s \varphi-a s(\varphi+\psi)-(l-a) s(\varphi+\psi+\theta) .
\end{aligned}
$$

By deriving the obtained expressions we have

$$
\begin{aligned}
\dot{x}= & {[-r s \varphi+a s(\varphi+\psi)+(l-a) s(\varphi+\psi+\theta)] \dot{\varphi} } \\
& +[a s(\varphi+\psi)+(l-a) s(\varphi+\psi+\theta)] \dot{\psi}+(l-a) s(\varphi+\psi+\theta) \dot{\theta}, \\
\dot{y}= & {[r c \varphi-a c(\varphi+\psi)-(l-a) c(\varphi+\psi+\theta)] \dot{\varphi} } \\
& -[a c(\varphi+\psi)+(l-a) c(\varphi+\psi+\theta)] \dot{\psi}+(l-a) c(\varphi+\psi+\theta) \dot{\theta} .
\end{aligned}
$$

The kinetic energy of the system is of the form then

$$
\begin{aligned}
T= & \frac{1}{2} J_{0} \dot{\varphi}^{2}+\{[-r s \varphi+a s(\varphi+\psi)+(l-a) s(\varphi+\psi+\theta)] \dot{\varphi} \\
& -[a s(\varphi+\psi)+(l-a) s(\varphi+\psi+\theta)] \dot{\psi}+(l-a) s(\varphi+\psi+\theta) \dot{\theta}\}^{2}+\frac{1}{2} m \dot{y}^{2} .
\end{aligned}
$$


The generalised forces corresponding to the coordinates $\varphi, \psi, \theta$ and $y$ respectively are of the form

$$
\begin{aligned}
Q_{\varphi}^{0} & =M+(N-P)[r c \varphi-l c(\varphi+\psi)], \\
Q_{\psi}^{0} & =F l s(\varphi+\psi)-(N-P) l c(\varphi+\psi), \\
Q_{\theta}^{0} & =F(l-a) s(\varphi+\psi)-(N-P)(l-a) c(\varphi+\psi), \\
Q_{y} & =N-P .
\end{aligned}
$$

Let us choose the quasi-accelerations to be $\ddot{\varphi}$ and $\ddot{y}$.

Following (2.7) and (2.8) the matrix $\mathbf{D}$ takes the form

where

$$
\mathbf{D}=\left[\begin{array}{llll}
1 & 0 & k_{\varphi} & 0 \\
0 & 1 & k_{y} & 0
\end{array}\right],
$$

Therefore

$$
\begin{aligned}
& k_{\varphi}=\frac{r c \varphi-a c(\varphi+\psi)-(l-a) c(\varphi+\psi+\theta)}{a c(\varphi+\psi)+(l-a) c(\varphi+\psi+\theta)}, \\
& k_{y}=\frac{1}{a c(\varphi+\psi)+(l-a) c(\varphi+\psi+\theta)} .
\end{aligned}
$$

It is easy to calculate the following quantities

$$
\frac{d}{d t} \frac{\partial T^{0}}{\partial \dot{\varphi}}-\frac{\partial T^{0}}{\partial \varphi}=J_{0} \ddot{\varphi},
$$

$$
\begin{aligned}
& \frac{d}{d t} \frac{\partial T^{0}}{\partial \dot{\psi}}-\frac{\partial T^{0}}{\partial \psi}=m\left[l s(\varphi+\psi) \ddot{\psi}-r s \varphi \dot{\varphi}^{2}+l c(\varphi+\psi)(\dot{\varphi}+\dot{\psi})^{2}\right] l s(\varphi+\psi), \\
& \frac{d}{d t} \frac{\partial T^{0}}{\partial \dot{\theta}}-\frac{\partial T^{0}}{\partial \theta}=m\left[l s(\varphi+\psi) \ddot{\psi}-r s \varphi \dot{\varphi}^{2}+l c(\varphi+\psi)(\dot{\varphi}+\dot{\psi})^{2}\right](l-a) s(\varphi+\psi) .
\end{aligned}
$$

By applying the equation (2.13) we obtain the equation of motion of the slider-crank mechanism, that is

$$
\begin{aligned}
& J_{0} \ddot{\varphi}-M-(N-P)[r c \varphi-l c(\varphi+\psi)]+m \frac{r c \varphi-l c(\varphi+\psi)}{l c(\varphi+\psi)}\{[l s(\varphi+\psi) \ddot{\psi} \\
& \left.\left.-r s \varphi \dot{\varphi}^{2}+l c(\varphi+\psi)(\dot{\varphi}+\dot{\psi})^{2}\right] l s(\varphi+\psi)-F l s(\varphi+\psi)+(N-P) l c(\varphi+\psi)\right\}=0 .
\end{aligned}
$$

By reducing the obtained result the equation of motion of the slider-crank mechanism will be then

$$
\begin{aligned}
& J_{0} \ddot{\varphi}-M+m \frac{r c \varphi-l c(\varphi+\psi)}{l c(\varphi+\psi)}\{[l s(\varphi+\psi) \ddot{\psi} \\
& \left.\left.-r s \varphi \dot{\varphi}^{2}+l c(\varphi+\psi)(\dot{\varphi}+\dot{\psi})^{2}\right] s(\varphi+\psi)-F s(\varphi+\psi)\right\}=0 .
\end{aligned}
$$


In order to determine the bending moment let us calculate the reaction force $R_{\theta}$, which is of the form

$$
R_{\theta}=\frac{d}{d t}\left(\frac{\partial T}{\partial \dot{\theta}}\right)^{0}-\left(\frac{\partial T}{\partial \theta}\right)^{0}-Q_{\theta}^{0}
$$

The expression of the bending moment will be then

$$
\begin{aligned}
R_{\theta}= & m\left[l s(\varphi+\psi) \ddot{\psi}-r s \varphi \dot{\varphi}^{2}+l c(\varphi+\psi)(\dot{\varphi}+\dot{\psi})^{2}\right](l-a) s(\varphi+\psi) \\
& +F(l-a) c(\varphi+\psi)+(N-P)(l-a) s(\varphi+\psi) .
\end{aligned}
$$

The reaction force $N$ is found by mean of equation (2.14) that is

$$
k_{y}^{0}\left(\frac{d}{d t} \frac{\partial T^{0}}{\partial \dot{\psi}}-\frac{\partial T^{0}}{\partial \psi}-Q_{\psi}^{0}\right)=0
$$

We have then

$$
\begin{aligned}
& m\left[l s(\varphi+\psi) \ddot{\psi}-r s \varphi \dot{\varphi}^{2}+l c(\varphi+\psi)(\dot{\varphi}+\dot{\psi})^{2}\right] l s(\varphi+\psi) \\
& +F l c(\varphi+\psi)+(N-P) l c(\varphi+\psi)=0 .
\end{aligned}
$$

This equation gives us:

$$
N=P-m\left[l s(\varphi+\psi) \ddot{\psi}-r s \varphi \dot{\varphi}^{2}+l c(\varphi+\psi)(\dot{\varphi}+\dot{\psi})^{2}\right] \frac{s(\varphi+\psi)}{c(\varphi+\psi)}+F
$$

It is easy to check the true of the obtained results. For this purpose let us consider the piece $B K$ (Fig. 4) and apply the D'Alembert method by putting the force of inertia of the slider $B$, which is equal to $-m \ddot{x}$. By using the equilibrium equation (the moment equation) for the set of force: $\vec{N}, \vec{P},-m \ddot{x}, M_{b}, N_{t}$ and $Q_{s h}$ we get

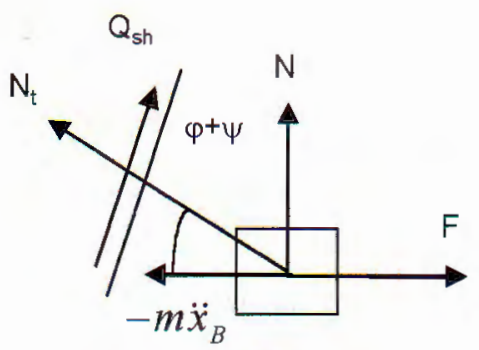

Fig. 4

$$
M_{b}=m \ddot{x}(l-a) s(\varphi+\psi)-F(l-a) s(\varphi+\psi)+(N-P)(l-a) c(\varphi+\psi),
$$

where $M_{b}$ is the bending moment, $\vec{N}_{t}$-the tensile force and $Q_{s h}$-the shear force.

The expression of $M_{b}$ is coincided with the one of the bending moment $R_{\theta}$ in (3.14) if taking into account the expression $\ddot{x}$ :

$$
\ddot{x}=l s(\varphi+\psi) \ddot{\psi}-r s \varphi \dot{\varphi}^{2}+l c(\varphi+\psi)(\dot{\varphi}+\dot{\psi})^{2} .
$$

Example 2. Let us determine the components of internal forces at the cross section as shown in the example 1. For this purpose let us make the system as in Fig. 5. 


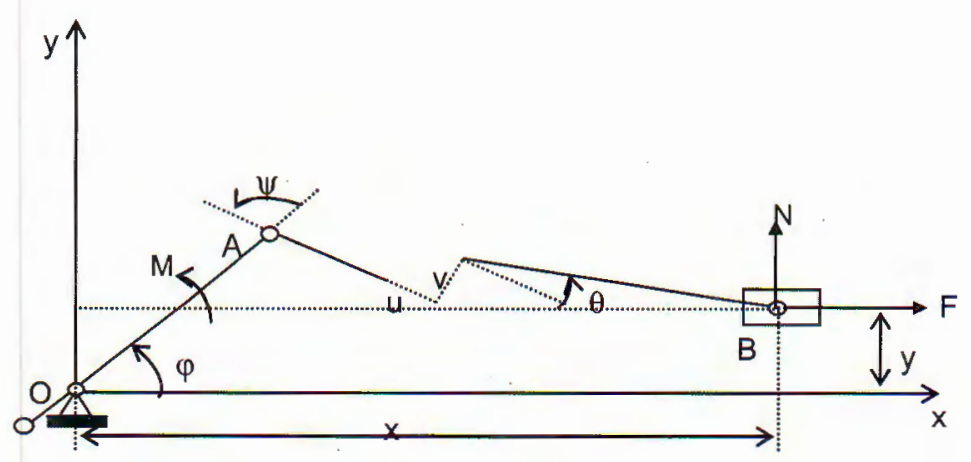

Fig. 5

The generalised coordinates are chosen to be $\varphi, \psi, u, v, \theta, y$ as in Fig. 5 .

The coordinates of the mass center of the slider are calculated by the expressions

$$
\begin{aligned}
& x=r c \varphi-(a+u) c(\varphi+\psi)+v s(\varphi+\psi)-(l-a) c(\varphi+\psi+\theta), \\
& y=r s \varphi-(a+u) s(\varphi+\psi)-v c(\varphi+\psi)-(l-a) s(\varphi+\psi+\theta) .
\end{aligned}
$$

We have then

$$
\begin{aligned}
\dot{x}= & {[-r s \varphi+(a+u) s(\varphi+\psi)+v c(\varphi+\psi)+(l-a) s(\varphi+\psi+\theta)] \dot{\varphi} } \\
& +[(a+u) s(\varphi+\psi)+v c(\varphi+\psi)-(l-a) s(\varphi+\psi+\theta)] \dot{\psi} \\
& -(l-a) s(\varphi+\psi+\theta) \dot{\theta}-\dot{u} c(\varphi+\psi)-\dot{v} s(\varphi+\psi), \\
\dot{y}= & {[r c \varphi-(a+u) c(\varphi+\psi)+v s(\varphi+\psi)-(l-a) c(\varphi+\psi+\theta)] \dot{\varphi} } \\
& -[(a+u) c(\varphi+\psi)-v s(\varphi+\psi)+(l-a) c(\varphi+\psi+\theta)] \dot{\psi} \\
& -(l-a) c(\varphi+\psi+\theta) \dot{\theta}-\dot{u} s(\varphi+\psi)-\dot{v} c(\varphi+\psi) .
\end{aligned}
$$

The kinetic energy of the system is of the form

$$
\begin{aligned}
T= & \frac{1}{2} J_{0} \dot{\varphi}^{2}+\frac{1}{2} m \dot{x}^{2}+\frac{1}{2} m \dot{y}^{2}=\frac{1}{2} J_{0} \dot{\varphi}^{2}+\frac{1}{2} m \dot{y}^{2} \frac{1}{2} m\{[-r s \varphi \\
& +(a+u) s(\varphi+\psi)+v c(\varphi+\psi)-(l-a) s(\varphi+\psi+\theta)] \dot{\varphi} \\
& +[(a+u) s(\varphi+\psi)+v c(\varphi+\psi)-(l-a) s(\varphi+\psi+\theta)] \dot{\psi} \\
& -(l-a) s(\varphi+\psi+\theta) \dot{\theta}-\dot{u} c(\varphi+\psi)+\dot{v} s(\varphi+\psi)\}^{2}
\end{aligned}
$$

The constraint equations will be

$$
\begin{aligned}
& y-r s \varphi+(a+u) s(\varphi+\psi)+v c(\varphi+\psi)+(l-a) s(\varphi+\psi+\theta)=0 \\
& u=0 \\
& v=0 \\
& \theta=0 .
\end{aligned}
$$


The generalised forces are of the form

$$
\begin{aligned}
& Q_{\varphi}^{0}=M+[r c \varphi-l c(\varphi+\psi)](N-P), \\
& Q_{\psi}^{0}=F l s(\varphi+\psi)-(N-P) l c(\varphi+\psi), \\
& Q_{u}^{0}=-F c(\varphi+\psi)-(N-P) s(\varphi+\psi), \\
& Q_{v}^{0}=-F s(\varphi+\psi)-(N-P) c(\varphi+\psi), \\
& Q_{\theta}^{0}=-F(l-a) s(\varphi+\psi)-(N-P)(l-a) c(\varphi+\psi) .
\end{aligned}
$$

In order to calculate the matrix $\mathbf{D}$ let us choose the quasi-accelerations to be $\ddot{\varphi}$ and $\ddot{y}$. The matrix $\mathbf{D}$ takes the form

$$
\mathbf{D}=\left[\begin{array}{llllll}
1 & 0 & k_{\varphi} & 0 & 0 & 0 \\
0 & 1 & k_{y} & 0 & 0 & 0
\end{array}\right]
$$

where the coefficients $k_{\varphi}$ and $k_{y}$ are of the form

$$
\begin{aligned}
& k_{\varphi}=\frac{r c \varphi-(a+u) c(\varphi+\psi)+v s(\varphi+\psi)-(l-a) c(\varphi+\psi+\theta)}{(a+u) c(\varphi+\psi)-v s(\varphi+\psi)+(l-a) c(\varphi+\psi+\theta)}, \\
& k_{y}=-\frac{1}{(a+u) c(\varphi+\psi)-v s(\varphi+\psi)+(l-a) c(\varphi+\psi+\theta)} .
\end{aligned}
$$

We have then

$$
k_{\varphi}^{0}=\frac{r c \varphi-l c(\varphi+\psi)}{l c(\varphi+\psi)}, \quad k_{y}^{0}=\frac{1}{l c(\varphi+\psi)} .
$$

It is easy to see that the relations (3.10), (3.11) and (3.12) are still true. Therefore the equation of motion is still of the from (3.13), the expression of the bending moment (3.14) and the reaction force of the slide-way acting on the slider (3.14).

In order to calculate the tensile and shear forces let us use first two equations of (2.15). For this purpose we calculate the following expressions:

$$
\begin{aligned}
& \frac{d}{d t}\left(\frac{\partial T}{\partial \dot{\psi}}\right)^{0}-\left(\frac{\partial T}{\partial \psi}\right)^{0}=-m\left[l s(\varphi+\psi) \ddot{\psi}-r s \varphi \dot{\varphi}^{2}+l c(\varphi+\psi)(\dot{\varphi}+\dot{\psi})^{2}\right] c(\varphi+\psi), \\
& \frac{d}{d t}\left(\frac{\partial T}{\partial \dot{v}}\right)^{0}-\left(\frac{\partial T}{\partial v}\right)^{0}=m\left[l s(\varphi+\psi) \ddot{\psi}-r s \varphi \dot{\varphi}^{2}+l c(\varphi+\psi)(\dot{\varphi}+\dot{\psi})^{2}\right] s(\varphi+\psi) .
\end{aligned}
$$

The tensile and shearing forces are calculated by the following formulas

$$
\begin{aligned}
R_{u}=\frac{d}{d t}\left(\frac{\partial T}{\partial \dot{u}}\right)^{0}-\left(\frac{\partial T}{\partial u}\right)^{0}-Q_{u}^{0}= & -m\left[l s(\varphi+\psi) \ddot{\psi}-r s \varphi \dot{\varphi}^{2}+l c(\varphi+\psi)(\dot{\varphi}+\dot{\psi})^{2}\right] c(\varphi+\psi) \\
& +F c(\varphi+\psi)+(N-P) s(\varphi+\psi), \\
R_{v}=\frac{d}{d t}\left(\frac{\partial T}{\partial \dot{v}}\right)^{0}-\left(\frac{\partial T}{\partial v}\right)^{0}-Q_{v}^{0}= & m\left[l s(\varphi+\psi) \ddot{\psi}-r s \varphi \dot{\varphi}^{2}+l c(\varphi+\psi)(\dot{\varphi}+\dot{\psi})^{2}\right] s(\varphi+\psi) \\
& +F s(\varphi+\psi)+(N-P) s(\varphi+\psi),
\end{aligned}
$$

where the reaction force $N$ takes the value of (3.15).

It is easy directly to check the true of the obtained results by means of the D'Alembert method. 


\section{Conclusion}

In the paper it is represented the method of determining the components of internal force at any cross section of the links of a closed chain. This method is a expanded form of the Lurie's method, which allows simultaneously to determine the reaction force of external constraints, the components of internal forces and the acceleration state of the system.

This publication is completed with financial support from the National Basic Research Program in Natural Sciences.

\section{References}

1. Do Sanh, Problem of determining reaction forces, $P M M, 6$ (1975) (in Russian).

2. Do Sanh, On the dynamic action of each constraint on a mechanical system, Nonlinear Vibration, No 20, 1979, Varsaw.

3. Do Sanh, The Motion of Constrained Mechanical Systems, The thesis of Science Doctor, Hanoi, 1984 (in Vietnamese).

4. Do Sanh, A Form of Equations of Motion of a Constrained Mechanical System, Proceeding of International Conference of Applied Dynamics, Hanoi (1995) 63-67.

5. Do Sanh, On the problem of first integral of mechanical systems, Proceedings of the National Conference "Vibration in Engineering" Hanoi 1 (2000) 174-185.

6. Gantmacher F., Lectures in Analytical Mechanics, Mir Publishers Moscow, 1970 (Translated from the Russian).

7. Kirghetov V. I., On the motion of a controlled mechanical system with conventional constraints (servo-constraints), Prikladnaja Mathematika i Mechanika 3 (1967), Moscow (in Russian).

8. Lurie A. I., Analytical Mechanics, GIFML, 1961 (in Russian).

Received October 25, 2003

\section{PHUUONG PHÁP XÁC ĐỊNH CÁC THÀNH PHẦN NỘI LỤC TA M MộT MặT CẮT NGANG CỦA THANH TRONG CO CẤU}

Trong bài báo đề xuất một phương pháp xác định các thành phần nội lực tại mặt cắt của thanh. Hiện nay vấn đề này được giải quyết nhờ phương pháp Đalămbe tiến hành theo hai giai đoạn, xác định trạng thái gia tốc và đặt lực quán tính, tiếp theo lập các phương trình cân bằng cho hệ lực gồm các lực đặt vào và lực quán tíṇ. Lurie $\mathrm{A}$. I. đã đề nghị một phương pháp dựa trên ý tưởng tạo nên một hệ mới bằng nhát cắt theo mặt cắt ngang. Để xác định vị trí của hệ bị cắt Lurie đã đưa vào các toạ độ phụ và đặt điều kiện là các toạ độ phụ phải bằng không. Điều này tương đương với việc đặt các liên kết lền hệ bị cắt để nó chính là hệ nguyên cũ. Phản lực các liên kết này chính là các thành phần nội lực tại mặt cắt.

Phương pháp của Lurie chỉ áp dụng thuận tiện cho các chuỗi mở, không áp dụng được các chuỗi đóng. Hơn nữa phương trình được sử dụng là phương trình Lagrang dạng nhân từ ít hiệu quà.

Trong bài báo đã xây dựng một phương pháp dựa trên ý tưởng của Lurie. Phương pháp này áp dụng cho cà chuỗi mở và chuỗi đóng nhờ áp dụng Nguyên lý phù hợp. 